\title{
Development of a model for the management of environmental safety of the region, taking into account of the GIS capacity
}

\author{
Viacheslav Burlov ${ }^{1 *}$, Andrey Andreev ${ }^{1}$ and Fedor Gomazov ${ }^{1}$ \\ ${ }^{1}$ Peter the Great St.Petersburg Polytechnic University, Polytechnicheskaya, 29, St. Petersburg, \\ 195251, Russia
}

\begin{abstract}
The system of space monitoring (SM) is of great importance, as a means of ensuring environmental safety. This system is based on remote sensing. The structure of SM is a distributed system. This system comprises independent data storage, system control, system of dynamic ratings, capacity and forecasting, control system, information system(IS) processing of monitoring data. As IS it is necessary to choose a geographic information system (GIS). IS monitoring refers to the problem-oriented system. These information systems include specialized databases models. All monitoring systems use sets of models, which allow building complex enterprise models. The peculiarity of the SM is the need to coordinate support of this monitoring and rate of the GIS capacity. Production Manager's decision is the impact on the object of monitoring. Results management and environmental data are received at the monitoring subsystem. Integration of SM and GIS monitoring has led to the creation of geoinformation space monitor (GISM). The operation of the system GISM is designed to provide a guaranteed result taking into account the capacity. Basis - the decision of the decision makers (DM). Therefore, an independent scientific and practical interest is the adequate mathematical model of DM.
\end{abstract}

\section{Introduction}

Ensuring environmental safety for geographically dispersed objects is based on the use of data from geoinformation systems. Receiving and processing data for GIS is the most important and time-consuming stage of creating such information systems. At present, the most promising and economically viable method is the method of obtaining data on objects based on remote sensing data (RS) and GPS measurements. Traditionally, remote sensing data refers only to those methods that make it possible to obtain an image of the earth's surface from space or from the air in any parts of the electromagnetic spectrum. Data from the GIS should be used to form a decision by the decision maker. At the same time, there arises the problem of establishing a connection between the GIS data and the decision model

\footnotetext{
* Corresponding author: burlovvg@mail.ru
} 
of the decision maker in the interests of ensuring the achievement of the goal of activities to ensure environmental safety. Without having the methodological basis for solving the tasks of managing environmental safety in the form of a condition for the existence of the process, we cannot guarantee the achievement of the goal of the activity.

The basis of activity is the person's decision. A person carries out his activity on the basis of the model [1-8]. Therefore, in order to implement an adequate road environment, it is necessary to have an adequate mathematical model for the solution of the person. In publications on the development of management decisions, it is asserted that it is very problematic to build a mathematical model of a person's decision. Although the publications presented only the results of the justification of the solution, but not the model of the solution itself. But without a mathematical model of solution, it is very difficult to guarantee the achievement of the goal of managing environmental safety (ES). The same situation with the construction of a system for ensuring environmental safety, as there is no criterion for the synthesis of a correctly constructed system. This set of factors determines the relevance of this work. And the goal is to select and justify the condition of the guaranteed achievement of the goal of managing environmental safety based on the synthesis of the mathematical model of the solution. Only the condition for the existence of the environmental safety management process will allow linking the data from GIS, obtained on the basis of remote sensing with elements of the mathematical model of the decision maker solution, into a single whole. Obtaining the condition for the existence of the environmental safety management process allows constructing a constructive technology. The technology for managing the ES process is the transformation of the information and activity resources of decision maker in the interest of achieving the goal of the activity.

\section{Setting the Research Task}

Naturally-scientific approach to the synthesis of the model of human solution. In the process of organizing and managing the ES process, there is often a situation where the results of decisions made do not justify the expectation of a person. An unsatisfactory result of management is justified by contradictory conclusions. To avoid contradictory conclusions, one should use the axiomatic method. Only this method allows excluding arbitrariness in reasoning. The axiomatic method assumes the existence of the following elements:

- Basic assumptions and assumptions, usually expressed in basic principles.

- Basic concepts, key words, axioms; Rules of inference; Theorems.

For objective use of this method, it should be noted that in the process of activities involved:

- A man, his consciousness.

- The world around (the object).

- Nothing that is given by nature and allows for cognition (Universal communication).

To create conditions that guarantee the achievement of the goal of the activity, the science based approach (SBA) to information security management is used. SBA is determined by the integration of the properties of human thinking, the surrounding world and cognition $[2,3,4]$. It is implemented by the scientific and pedagogical school "System Integration of Public Administration Processes" [5]. Three-component is reflected in three principles:

1. The principle of three-component knowledge:

- Component A. Abstractrepresentation (condition of existence, methodology);

- Component B. Abstract-specific (cause-effect relationships, methods); 
- Component C. Concrete representation (technology, algorithms).

2. Principle of the integrity of the world. The law of preserving the integrity of the object (LPIO) [9-11] is implemented. This is a stable, objective, repetitive relationship between the properties of an object and an action for a fixed purpose.

3. The principle of the know ability of the world. It is implemented by methods. Decomposition. Abstraction. Aggregation.

The principle of the three-component cognition is that a person, consciously or not, is making a decision in three levels to represent the situation. 1 - Abstract level (the condition for the existence of the process is formed). 2 - abstract-concrete level (causal relationships are formed). 3 - Specific (conditions for the realization of cause-effect relationships are formed).

In the process of activity, a person operates with the categories "system", "model" and "destination". Therefore, it is especially correct to consider and use these categories.

There are only two directions of system development (models) - development of the system on the basis of analysis and development on the basis of synthesis. This approach is known from the monograph on system engineering [13]. Anokhin P.K. pointed out and experimentally confirmed that for the synthesis of the system it is necessary to reveal the "basic regularity" of the general theory of functional systems [8]. Including appealing to leading specialists in the field of creation and research (for example, to M. Mesarovic [14]) with the question of developing a formalized criterion for constructing a system. However, this question was not answered in well-known publications, but is being developed by the scientific and pedagogical school "System Integration of Public Administration Processes" in the form of the law of preserving the integrity of the facility [12]. Therefore, in the present work, LPIO is used to synthesize the solution model [9-11].

Also it is necessary to consider the feature of synthesizing the model of the object (process). The key moment is the condition of its adequacy. The most acceptable approach for assessing adequacy is the "completeness of taking into account the basic laws of the domain".

If the laws of physics, chemistry are used in the field of natural sciences for the adequacy of the model being developed. Those areas of complex systems, social, economic, technical and technological systems, and others are encouraged to use the law of conservation of integrity [9-11].

General approach to the synthesis of the solution model in the management of environmental safety. The basis for the management of ES is always the decision of decision maker. The person takes on the basis of the model. Under the model of an object we mean a description or representation of an object corresponding to an object and allowing obtaining characteristics about this object. Therefore, the solution is the model of the process with which the person works. A process is an object in operation with a fixed destination. For synthesis, we use SBA, based on LPIO.

The law of preserving the integrity of an object is a stable repetitive link between the properties of an object and the properties of an action for a fixed purpose. LPIO is manifested in the mutual transformation of the properties of the object and the properties of its action for a fixed purpose.

In accordance with the SBA developed, each process should be represented by three components, corresponding to the properties of "objectivity", "integrity" and "variability" (or concepts "object", "purpose" and "action" respectively). These three components are arranged horizontally. On the one hand, they can be interpreted in three different levels of knowledge of the world (abstract, abstract-concrete, concrete). This approach determines the presence of three levels along the vertical. Figure 1 presents a structural diagram of the 
deployment of the content of the concept "Solution" [15]

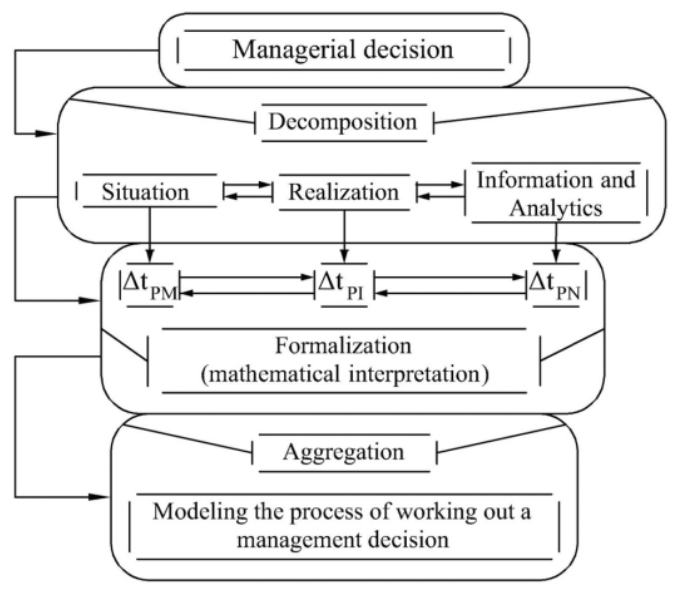

Fig. 1. The schematic structure of management decision acquisition

Management technology represents agoal-directed sequence ofmanagement operations, as a result of which a decision is made and realized. The management technology is modeled with the help of project networks and operation cards of the management process.

The functions of management are the product of specialization and systematization of labor. Each represents a part of the management process and is identified based on some definite feature. A distinction is made between main functions (prognostics, planning, standardization, accounting, control, analysis, regulation or coordination) and particular functions (for example, human resource management).

The management system has a hierarchical structure: according to the "vertical" specialization of labor, separate levels of management such as top, middle and lower management are distinguished. [16]

The structure of management is the structure of a management object, considering the requirements of the best functioning the system.

Management decision is the bas is ofstaffmanagement. The managerial decision is a logical interrelation between the levels of management and functional areas created in a form that allows achieving the goals in the most efficient way.

The process of decision-making involves: setting a goal; collecting and processing information; preparing draft decisions; coordination; making a decision; control.

The analys is of the existing management systems revealed a number of dis-advantages in capacity of GIS:

- the indicators of management process es monitoring are either not defined, inadequate, or do not provide any realopportunity for a full and comprehensive as sessment of the process;

- monitoring and assessment of the management processes are just formalities or lacking altogether;

- the results of monitoring are not used to evaluate tendencies, analyze situations or make management decisions on corrective or preventive actions with the purpose of continual improvement;

- analysis of information streams of control bodies shows that today only $10-15 \%$ of the available information is used for making management decisions;

- only $6-10 \%$ of the available information is used in the function of planning and 
prognostics;

- only $25-30 \%$ of the available information is used in the function of control. Studying the current concept of GIS capacity on the basis of analysis showed that a goal is achieved by gradual task performance, and at every stage the manager coordinates resources for the performance, which leads to the following drawbacks:

- greater computational resources required for the optimization of plans;

- more time required for coordinating resources;

- more time required for the manager to decide.

It is necessary to exclude the use of verbal models, take into consideration the logic of the way the management systemoperates, and reduce the time the manager requires to decide on GIS capacity based on automatic control. [17]

To exercise automatic control, it is necessary to get the conditions of a process' existence and provide feedback. [18]

To that effect, the concept of GIS capacity needs to be changed. Figure 2 shows the approach to maintaining the functioning of a GIS capacity based on feedback.

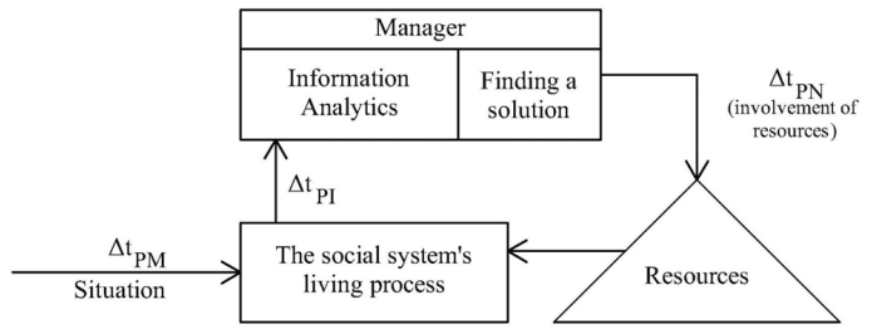

Fig. 2. The conceptions of maintaining the GIS cap acity functioning on the feedback basis

Synthesis of the model of environmental safety management as a result of the application of decomposition, abstraction and aggregation methods, was transformed the concept of "management decision" into an aggregate - a mathematical model of the management solution of the following kind.

\section{Model Development}

When the object (process) of management is in state 3, the given problem needs to be identified. Naturally, the manager spends some time $\Delta t_{P I}$ on the problem's identification. At this stage, preparations for involving additional resources for the problem's solution take place. Thus, during the solution analysis the managed system transits into state 4 , where the manager understands what resources should be used for the achievement of the management goal and how. In this state, there are two variants of the GIS capacity development: [9]

- Capacity is not ready to solve the problem. In this situation the system moves from state 4 into state 1 (the initial state) - the problem of capacity is not solved;

- Capacity can solve the problem, but it takes some time, so he moves from state 4 into state 2, i.e. the problem is solved.

There are two possible scenarios of situation development in state 2:

- Capacity spent unacceptably too much time solving the problem, which is equivalent to unfulfilling the target task in the management process. This is characterized by moving from state 2 into state 1 ;

- Capacity on the problem's solution is within acceptable limits when the manager solves the management target task. 
The system transition back into its initial state characterizes the capacity on a great number of problems. The frequency of the system's transition from state 1 into state $2\left(\xi^{+}\right)$ is equivalent to the value reciprocal of the average time of fulfilling the target task, which characterizes the level of the GIS capacity for solving the target tasks, while the frequency $(\xi$ -) characterizes the average frequency of unfulfilling the target task, but usually this value must vary within $0,1 \%$.

The frequency of transition from state 4 into state 2 is the value $v_{2}=\frac{1}{\Delta t_{P N}}$ being the average time of the problem's neutralization. The level of competence for solving unknown tasks depends on the correlation $v_{2}$.

The frequency of transition from state 4 into state 1 is the quantity $v_{3}$. - the frequency of the problem's solution breakdown by the manager because of his inability to identify the situation (the indicator of managerial qualification). The indicator of managerial qualification depends on the frequency $v_{3}$. [19]

This logic of reasoning allows us to construct the following graph (Fig. 3)

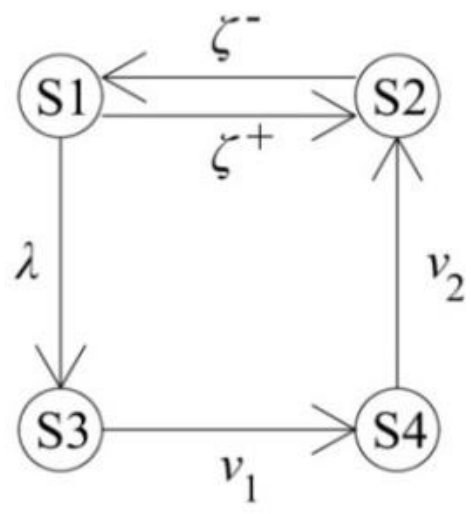

Fig. 3. The state graph

The main assumptions and presumptions:

1) The information capacity is being considered.

2) Time intervals between the moments of problem manifestation detection are random values.

3) The detected facts form a stream in time that is closely approximated to Pois son arrival.

4) The time for processing the data on a desired feature is a random value.

5) The data processed within the system is further shared between the allocated forces and means, solving the corresponding target tasks in the production control.

6) The instance is being considered when the stay period of the desired features (facts) in the sphere of production capacity systemis extremely limited, comparable to the time that is necessary for them to be detected as well as for data to be processed and adequate measures to be taken according to these features.

7) The systemunder discussion is meant for evaluating the potentialopportunities for the production capacity system, depending on the created situation.

Against the background, Kolmogorov differential equation system can be used:

$$
\frac{d P_{i}(t)}{d t}=\sum_{j=1}^{n} \lambda_{j i}(t) * P_{j}(t)-P_{i}(t) * \sum_{j=1}^{n} \lambda_{j i}(t)
$$

where $\mathrm{i}=0,1,2, \ldots, \mathrm{n}$.

To produce a Kolmogorov differential equation for the function $P_{i}(t), \mathrm{i}=0,1,2, \ldots, \mathrm{n}$, the derivative $\frac{d P_{i}(t)}{d t}$ of the function $P_{i}(t)$ must be written in the left part of this equation, while 
in the right part of the equation there must be the product $\left(\sum_{j=1}^{n} \lambda_{j i}\right) P_{i}(t)$ of the $\operatorname{sum} \sum_{j=1}^{n} \lambda_{j i}$ of transition probability densities $\lambda_{j i}$ with arrows coming out of state $S_{i}$, by the probability $P_{i}(t)$ of this state marked "minus", plus the sum $\left(\sum_{j=1}^{n} \lambda_{j i}\right) P_{j}(t)$ of the products $\lambda_{j i} P_{j}(t)$ of transition probability densities $\lambda_{j i}$, corresponding to the arrows coming into state $\mathrm{S}_{\mathrm{i}}$, by the probabilities of the states $P_{j}(t)$ where these arrows come out from. Herein the transition probability densities $\lambda_{j i}$ corresponding to the arrows absent on the graph equal zero. [9]

Final probabilities of states can be calculated by solving the system of linear algebraic equations that are obtained from Kolmogorov differential equations if derivatives equal zero and the probability functions of states $P_{1}(t), \ldots, P_{n}(t)$ in the right parts of the equations transit correspondingly into the unknown final probabilities $\mathrm{P}_{1}, \ldots, \mathrm{P}_{\mathrm{n}}$. To find the exact value $\mathrm{P}_{1}, \ldots, \mathrm{P}_{\mathrm{n}}$, the normalizing condition $P_{0}+P_{1}+\cdots+P_{n}=1$ is added to the equations.

Let us produce a system of Kolmogorov equations for the state graph in Fig. 3

$$
\left\{\begin{array}{l}
\frac{d P_{1}(t)}{d t}=-\left(\xi^{+}+\lambda\right) * P_{1}(t)+\xi^{-} * P_{2}(t) \\
\frac{d P_{2}(t)}{d t}=\xi^{+} * P_{1}(t)-\xi^{-} * P_{2}(t)+v_{2} * P_{4}(t) \\
\frac{d P_{3}(t)}{d t}=\lambda * P_{1}(t)-v_{1} * P_{3}(t) \\
\frac{d P_{4}(t)}{d t}=v_{1} * P_{3}(t)-v_{2} * P_{4}(t)
\end{array}\right.
$$

Then the final probabilities can be calculated by solving a systemof linear algebraic equations.

$$
\left\{\begin{array}{l}
0=-\left(\xi^{+}+\lambda\right) * P_{1}+\xi^{-} * P_{2} \\
0=\xi^{+} * P_{1}-\xi^{-} * P_{2}+v_{2} * P_{4} \\
0=\lambda * P_{1}-v_{1} * P_{3} \\
1=P_{1}+P_{2}+P_{3}+P_{4}
\end{array}\right.
$$

The systemsolution is as follows:

$$
\begin{aligned}
P_{1} & =\frac{v_{1} * v_{2} * \xi^{-}}{\lambda * v_{1} * v_{2}+\lambda * v_{1} * \xi^{-}+\lambda * v_{2} * \xi^{-}+v_{1} * v_{2} * \xi^{+}+v_{1} * v_{2} * \xi^{-}} \\
P_{2} & =\frac{\lambda * v_{1} * v_{2}+v_{1} * v_{2} * \xi^{+}}{\lambda * v_{1} * v_{2}+\lambda * v_{1} * \xi^{-}+\lambda * v_{2} * \xi^{-}+v_{1} * v_{2} * \xi^{+}+v_{1} * v_{2} * \xi^{-}} \\
P_{3} & =\frac{\lambda * v_{2} * \xi^{-}}{\lambda * v_{1} * v_{2}+\lambda * v_{1} * \xi^{-}+\lambda * v_{2} * \xi^{-}+v_{1} * v_{2} * \xi^{+}+v_{1} * v_{2} * \xi^{-}} \\
P_{4} & =\frac{\lambda * v_{1} * \xi^{-}}{\lambda * v_{1} * v_{2}+\lambda * v_{1} * \xi^{-}+\lambda * v_{2} * \xi^{-}+v_{1} * v_{2} * \xi^{+}+v_{1} * v_{2} * \xi^{-}}
\end{aligned}
$$

The probability of the problem being identified and neutralized by the capacity is determined by the following correlation:

$$
P_{2}=\frac{\lambda * v_{1} * v_{2}+v_{1} * v_{2} * \xi^{+}}{\lambda * v_{1} * v_{2}+\lambda * v_{1} * \xi^{-}+\lambda * v_{2} * \xi^{-}+v_{1} * v_{2} * \xi^{+}+v_{1} * v_{2} * \xi^{-}}
$$

\section{Conclusion}

In general, the work suggests the basics of building ES management technology. The synthesis of the ES control system based on a system of differential equations allowed to realize a guaranteed approach to ES management. The controlmodel, the state graph, can be further complicated by introducing additional feedbacks and taking into account other conditions. 


\section{References}

1. V. G. Bondur, A. I. Savin. Study of Earth from space. No. 6. C. p 70 - 78. (1992)

2. E. A. Lupyan and others. Modern problems of remote sensing of the Earth from space. Vol. 8. No. 1. P. 26-43 (2011)

3. V. Y. Tsvetkov. The Development of problem-oriented control system SCST, Vnticentr, 131 p. (1991)

4. V. Y. Tsvetkov, V. T. Matchin. Slavic forum. 2(6) p. 77-81. (1991)

5. V. G. Bondur, K. Ya. Kondratyev, V. F. Krapivin, V. P. Savinykh. Earth exploration from space. No. 1. p. 3-14. (2005)

6. V. M. Egorov, V. Ya. Tsvetkov. No. 4. p. 34-37 (2012)

7. N. N. Moiseev. Mathematical problems of system analysis. Nauka, Moscow, 468 p. (1981)

8. P. K. Anokhin. Systemic mechanisms of highernervous activity. Nauka, 453 p. (1979)

9. V. G. Burlov. Foundations of simulation of socio-economic and political processes (Methodology. Methods) Faculty of Complex Safety St. Petersburg state Polytechnical University. 265 p. (2007)

10. V. G. Burlov. Mathematical methods of modeling in Economics. St. Petersburg state Polytechnical University, Faculty of security, the NP "strategy for the future", V1 330 p. (2007)

11. V. G. Burlov. Information technology and systems: management, economy, transport, the right. No. 2 (16). P. 99-111. (2015)

12. The register of leading scientific and teaching schools of St. Petersburg. http://is.ifmo.ru/aboutus/2013/science-schools.pdf

13. G. H. Hood, R. E. Makol. Engineering Sov. Radio, 384 p. (1962)

14. M. D. Mesarovic, Yasuhiko Takahara. General Systems Theory: Mathematical Foundations Academic press New York, San Francisco, London (1975)

15. V. G. Burlov, O.M. Lepeshkin. Transportation Research Procedia, 20, p. 94-99 (2017)

16. V. G. Burlov, N.N. Popov. Advances in the Astronautical Sciences, 161, p. 751760 (2017)

17. V. G. Burlov, M. I. Grachev. Transportation Research Procedia, 20, p. 100-106 (2017)

18. S.S. Sokolov, O. M. Alimov, M. G. Golubeva, V. G. Burlov, N. M. Vikhrov. ElConRus, January, p. 124-127, (2018)

19. E. P. Istomin, V. M. Abramov, A. A. Fokicheva, A. G. Sokolov, V. G. Burlov. SGEM, 17(21), c. 1005-1012. (2017) 